
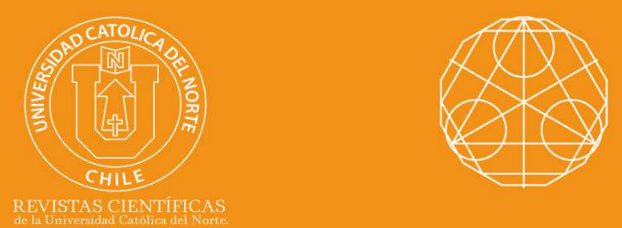

\title{
The edge-to-edge geodetic domination number of a graph
}

J. John ${ }^{1}$ (1) orcid.org/0000-0001-5528-4387

V. Sujin Flower²® orcid.org/0000-0002-3702-6875

${ }^{1}$ Government College of Engineering, Dept. of Mathematics, Tirunelveli, TN, India. -john@gcetly.ac.in

${ }^{2}$ Holy Cross College (Autonomous), Dept. of Mathematics, Nagercoil, TN, India.

v sujinflower@gmail.com

Received: 28 March 2020 | Accepted: 12 January 2021

\section{Abstract:}

Let $G=(V, E)$ be a connected graph with at least three vertices. $A$ set $S \subseteq E(G)$ is called an edge-to-edge geodetic dominating set of $G$ if $S$ is both an edge-to-edge geodetic set of $G$ and an edge dominating set of $G$. The edge-to-edge geodetic domination number $\gamma_{g e e}(G)$ of $G$ is the minimum cardinality of its edge-to-edge geodetic dominating sets. Some general properties satisfied by this concept are studied. Connected graphs of size $m$ with edge-to-edge geodetic domination number 2 or $m$ or $m-1$ are characterized. We proved that if $G$ is a connected graph of size $m \geq 4$ and $\bar{G}$ is also connected, then $4 \leq \gamma_{\text {gee }}(G)+\gamma_{\text {gee }}(\bar{G}) \leq 2 m-2$. Moreover we characterized graphs for which the lower and the upper bounds are sharp. It is shown that, for every pair of positive integers $a, b$ with $2 \leq a \leq b$, there exists a connected graph $G$ with $g_{e e}(G)=a$ and $\gamma_{g e e}(G)=b$. Also it is shown that, for every pair of positive integers a and $b$ with $2<a \leq b$, there exists a connected graph $G$ with $\gamma_{e}(G)=a$ and $\gamma_{g e e}(G)=b$, where $\gamma_{e}(G)$ is the edge domination number of $G$ and $g_{e e}(G)$ is the edge-to-edge geodetic number of $G$.

Keywords: Edge-to-edge geodetic domination number; Edge-to-edge geodetic number; Edge domination number; Domination number; Geodetic number

MSC (2020): 05C69, 05C12.

\section{Cite this article as (IEEE citation style):}

J. John and V. Sujin Flower, "The edge-to-edge geodetic domination number of a graph", Proyecciones (Antofagasta, On line), vol. 40, no. 3, pp. 635-658, 2021, doi: 10.22199/issn.0717-6279-4057

Article copyright: (C) 2021 J. John and V. Sujin Flower. This is an open access article distributed under the terms of the Creative Commons License, which permits unrestricted use and distribution provided the original author and source are credited. 


\section{Introduction}

By a graph $G=(V, E)$, we mean a finite, undirected connected graph without loops or multiple edges. The order and size of $G$ are denoted by $n$ and $m$ respectively. For basic graph theoretic terminology, we refer to [9]. Two vertices $u$ and $v$ of said to be adjacent in $G$ if $u v \in E(G)$. The neighborhood $N(v)$ of the vertex $v$ in $G$ is the set of vertices adjacent to $v$. The degree of the vertex $v$ is $\operatorname{deg}(v)=|N(v)|$. If $e=\{u, v\}$ is an edge of a graph $G$ with $\operatorname{deg}(u)=1$ and $\operatorname{deg}(v)>1$, then we call $e$ an end edge, $u$ a leaf and $v$ a support vertex. For any connected graph $G$, a vertex $v \in V(G)$ is called a cut vertex of $G$ if $V(G)-v$ is disconnected. The subgraph induced by set $S$ of vertices of a graph $G$ is denoted by $\langle S\rangle$ with $V(\langle S\rangle)=S$ and $E(\langle S\rangle)=\{u v \in E(G): u, v \in S\}$. A vertex $v$ is called an extreme vertex of $G$ if $\langle N(v)\rangle$ is complete. An edge $e$ is called an extreme edge of $G$ if one end of eis an extreme vertex of $G$. Let $c(G)$ be the length of the longest cycle in $G$. Let $\lceil x\rceil$ denotes the smallest integer greater than or equal to $x$.

A set of vertices $D$ in a graph $G$ is called a dominating set of $G$ if each vertex of $V(G)-D$ is adjacent to some vertex of $G$. The domination number $\gamma(G)$ of $G$ is the minimum cardinality of a dominating set of $G$. For references on domination parameters in graphs see $[9,11,12,13,19,20,26]$. Two edges are adjacent if they have a common vertex. A set of edges $S$ of $G$ is called an edge dominating set of $G$ if every edge of $E(G)-S$ is adjacent to an element of $S$. The edge domination number $\gamma_{e}(G)$ of $G$ is the minimum cardinality of an edge dominating set of $G$. An edge dominating set of cardinality $\gamma_{e}(G)$ is called a $\gamma_{e^{-}}$set of $G$. An independent edge set of a graph $G$ is a subset of the edges such that no two edges in the subset share a vertex of $G$. An edge dominating set $S$ is said to be an independent edge dominating set of $G$ if $S$ is an independent edge set of $G$. The edge domination number was studied in $[2,3,8,16,17,18,23,28]$.

A vertex $x$ is an internal vertex of an $u-v$ path $P$ if $x$ is a vertex of $P$ and $x \neq u, v$. An edge $e$ of $G$ is an internal edge of an $u-v$ path $P$ if $e$ is an edge of $P$ with both of its ends are in $P$. The distance $d(u, v)$ between two vertices $u$ and $v$ in a connected graph $G$ is the length of a shortest $u-v$ path in $G$. An $u-v$ path of length $d(u, v)$ is called an $u-v$ geodesic. A vertex $x$ is said to lie on an $u-v$ geodesic $P$ if $x$ is a vertex of $P$ including the vertices $u$ and $v$. For a vertex $v$ of $G$, the eccentricity $e(v)$ is the distance between $v$ and a vertex farthest from $v$. The minimum eccentricity among the vertices of $G$ is the radius, $\operatorname{rad}(G)$ and the maximum eccentricity is its diameter, $\operatorname{diam}(G)$. We denote $\operatorname{rad}(G)$ by $r$ and $\operatorname{diam}(G)$ by $d$. A star is a tree with 
diameter 2. A double star is a tree with diameter 3. The closed interval $I[u, v]$ consists of $u, v$ and all vertices lying on some $u-v$ geodesic of $G$. For a non-empty set $S \subseteq V(G)$, the set $I[S]=\cup_{u, v \in S} I[u, v]$ is the closure of $S$. A set $S \subseteq V(G)$ is called a geodetic set if $I[S]=V(G)$. Thus every vertex of $G$ is contained in a geodesic joining some pair of vertices in $S$. The minimum cardinality of a geodetic set of $G$ is called the geodetic number of $G$ and is denoted by $g(G)$. A geodetic set of minimum cardinality is called $g$-set of $G$. For references on geodetic parameters in graphs see $[4,5,6,7,10,14,15,22,23]$.

Let $G=(V, E)$ be a connected graph with at least three vertices. For subsets $A$ and $B$ of $V(G)$, the distance $d(A, B)$ is defined as $d(A, B)=$ $\min \{d(x, y): x \in A, y \in B\}$. An $u-v$ path of length $d(A, B)$ is called an $A$ - $B$ geodesic joining the sets $A$ and $B$, where $u \in A$ and $v \in B$. A vertex $x$ is said to lie on an $A$-B geodesic if $x$ is a vertex of an $A$ - $B$ geodesic [21]. A set $S \subseteq E(G)$ is called an edge-to-vertex geodetic set if every vertex of $G$ is either incident with an edge of $S$ or lies on a geodesic joining a pair of edges of $S$. The edge-to-vertex geodetic number $g_{e v}(G)$ of $G$ is the minimum cardinality of its edge-to-vertex geodetic sets and any edge-tovertex geodetic set of cardinality $g_{e v}(G)$ is called an $g_{e v}$-set of $G$. The edge-to-vertex geodetic number of a graph was studied in $[21,24,25,27]$. A set $S \subseteq E(G)$ is called an edge-to-edge geodetic set of $G$ if every edge of $G$ is an element of $S$ or lies on a geodesic joining a pair of edges of $S$. The edge-to-edge geodetic number $g_{e e}(G)$ of $G$ is the minimum cardinality of its edge-to-edge geodetic sets and any edge-to-edge geodetic set of cardinality $g_{e e}(G)$ is said to be a $g_{e e}$-set of $G$. This concept was studied in [1].

For $G=C_{5}$ with vertex set $V(G)=\left\{v_{1}, v_{2}, v_{3}, v_{4}, v_{5}\right\}, S_{1}=\left\{v_{1} v_{2}, v_{1} v_{5}, v_{3} v_{4}\right\}$ is a $g_{e e}$-set of $G$ and $S_{2}=\left\{v_{1} v_{2}, v_{4} v_{5}\right\}$ is a $\gamma_{e}$-set of $G$ so that $g_{e e}(G)=3$ and $\gamma_{e}(G)=2$. For $G=C_{8}$ with vertex set $V(G)=\left\{v_{1}, v_{2}, v_{3}, v_{4}, v_{5}, v_{6}, v_{7}, v_{8}\right\}$, $S_{3}=\left\{v_{1} v_{2}, v_{5} v_{6}\right\}$ is a $g_{e e}$-set of $G$ and $S_{4}=\left\{v_{1} v_{2}, v_{4} v_{5}, v_{7} v_{8}\right\}$ is a $\gamma_{e}$-set of $G$ so that $g_{e e}(G)=2$ and $\gamma_{e}(G)=3$. From this two examples we observed that $S_{2}$ is a $\gamma_{e}$-set of $G$ but not $g_{e e^{-s e t}}$ of $G$ and $S_{3}$ is a $g_{e e^{-s e t}}$ of $G$ but

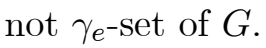

Domination in graphs has applications to several fields [20]. Domination arises in facility location problems, where the number of facilities (e.g., hospitals, fire stations) is fixed and one attempts to minimize the distance that a person needs to travel to get to the closest facility. Concepts related to geodesics also appear in the social sciences [7]. When members of a social group are represented by vertices, and particular relationships between members by edges of a graph, then the geodetic number can be considered as the smallest number $n$ such that every member of a group 
is contained in a minimal chain of relationships between chosen $n$ members. Edge domination in graphs has some very interesting applications in a telephone switching network [28]. A telephone switching network built to route phone calls from incoming lines to outgoing trunks (we assume that a trunk can pass only one phone call at a time). The problem is to find the worst-case behavior of the network, i.e., the minimum number of routed calls when the network is saturated and no calls can be added. For this we construct a bipartite graph $G$ by connecting a line to a trunk if and only if the line can be switched to the trunk. Then the problem is equivalent to finding the size of a minimum independent edge dominating set of $G$. The edge-to-vertex geodetic concepts have many applications in location theory and convexity theory. There are interesting applications of these concepts to the problem of designing the route for a shuttle and communication network design [21]. In the case of designing the route for a shuttle, although all the vertices are covered by the shuttle when considering edgeto-vertex geodetic sets, some of the edges may be left out. This drawback is rectified in the case of edge-to-edge geodetic sets and hence considering edge-to-edge geodetic sets is more advantageous to the real life application of routing problem. In particular, the edge-to-edge geodetic sets are more useful than edge-to-vertex geodetic sets in the case of regulating and routing the goods vehicles to transport the commodities to important places. This is the motivation behind the study of edge-to-edge geodetic concepts. An edge-to-edge geodetic set can be used to improve the effectiveness in networks. Throughout the following $G$ denotes a connected graph with at least three vertices.

\section{The edge-to-edge geodetic domination number of a graph}

It is seen that an edge dominating set is not in general an edge-to-edge geodetic set in a graph $G$. Also the converse is not valid in general. This has motivated us to study the new edge domination conception of edgeto-edge geodetic domination. We investigate those subsets of edges of a graph that are both an edge-to-edge geodetic set and an edge dominating set. We call these sets as edge-to-edge geodetic dominating sets. In this section, we look closely at the concept of edge-to-edge geodetic domination in a graph $G$, and obtain the edge-to-edge geodetic domination number of some families of graphs. Although the edge-to-edge geodetic domination number is greater than or equal to the edge-to-edge geodetic number for an arbitrary graph, the properties of the edge-to-edge geodetic geodetic 
dominating sets and results regarding edge-to-edge geodetic domination number are quite different from that of edge-to-edge geodetic concepts.

Definition 2.1. Let $G=(V, E)$ be a connected graph with at least three vertices. A set $S \subseteq E(G)$ is called an edge-to-edge geodetic dominating set of $G$ if $S$ is both an edge-to-edge geodetic set and an edge dominating set. The edge-to-edge geodetic domination number $\gamma_{g e e}(G)$ of $G$ is the minimum cardinality of its edge-to-edge geodetic dominating sets and any edge-to-edge geodetic dominating set of minimum cardinality is said to be a $\gamma_{g e e}$-set of $G$.

Example 2.2. For the graph $G$ given in Figure 2.1, $\gamma_{e}(G)=2, g_{e e}(G)=2$ and $S_{1}=\left\{v_{1} v_{2}, v_{3} v_{4}, v_{5} v_{6},\right\}, S_{2}=\left\{v_{1} v_{2}, v_{1} v_{3}, v_{5} v_{6}\right\}, S_{3}=\left\{v_{1} v_{2}, v_{2} v_{3}, v_{5} v_{6}\right\}$ and $S_{4}=\left\{v_{1} v_{2}, v_{4} v_{5}, v_{5} v_{6}\right\}$ are the only four $\gamma_{g e e}$-sets of $G$ so that $\gamma_{g e e}(G)=$ 3 .

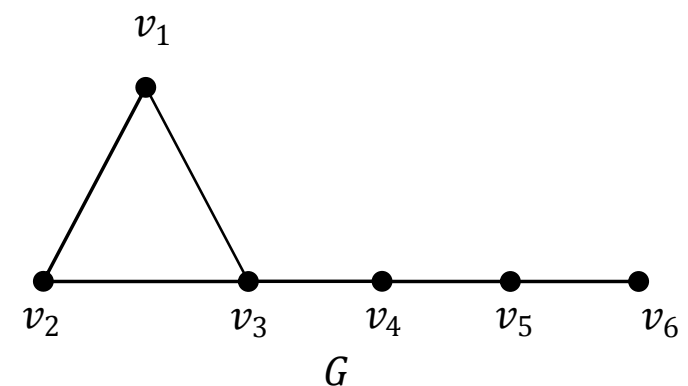

Figure 2.1

Observation 2.3. (i) Each end edge of a connected graph $G$ belongs to every edge-to-edge geodetic dominating set of $G$.

(ii) If $G$ is a star $K_{1, m}$ with size $m \geq 2$, then $\gamma_{\text {gee }}(G)=m$.

(iii) If $G$ is a double star with size $m \geq 3$, then $\gamma_{\text {gee }}(G)=m-1$.

(iv) If $G$ is a complete bipartite graph $K_{r, s}(2 \leq r \leq s)$, then $\gamma_{g e e}(G)=s$.

(v) If $G$ is a complete graph $K_{n}(n \geq 4)$, then $\gamma_{\text {gee }}(G)=\left\{\begin{array}{l}\frac{n}{2} ; \text { if } n \text { is even } \\ \frac{n+1}{2} ; \text { if } n \text { is odd }\end{array}\right.$

(vi) If $G$ is a cycle $C_{n}(n \geq 4)$, then $\gamma_{\text {gee }}(G)=\left\{\begin{array}{l}3 \text {; if } n=5 \\ \left\lceil\frac{n}{3}\right\rceil \text {; if } n \neq 5\end{array}\right.$ 


\section{Some results on the edge-to-edge geodetic domination num- ber of a graph}

In this section, we look at some relationships between the edge-to-edge geodetic domination number and other parameters. Further some improved upper bounds for edge-to-edge geodetic domination number of a graph are given.

Observation 3.1. If $G$ is a connected graph with size $m \geq 2$, then $2 \leq$ $\max \left(\gamma_{e}(G), g_{e e}(G)\right) \leq \gamma_{g e e}(G) \leq m$.

Theorem 3.2. Let $G$ be a connected graph with size $m \geq 2$. If $\gamma_{\text {gee }}(G)=$ 2 , then $d(e, f) \leq 2$ for every $e, f \in E(G)$.

Proof. Let $S=\{e, f\}$ be a $\gamma_{g e e}$-set of $G$. Let us assume that $e=u v$, $f=u^{\prime} v^{\prime}$ and $e-f$ geodesic $P$ be $u=u_{0}, u_{1}, u_{2}, \ldots, u_{n}=u^{\prime}$, where $u_{0}$ is either $u$ or $v$ and $u_{n}$ is either $u^{\prime}$ or $v^{\prime}$. On the the contrary assume that $d(e, f) \geq 3$ for some $e, f \in E(G)$. Which implies $n \geq 3$. Then the edge $u_{1} u_{2} \in E(G)-S$ is not dominated by any edge of $S$. Hence $S$ is not a geodetic dominating set of $G$, which is a contradiction. Therefore $d(e, f) \leq 2$ for every $e, f \in E(G)$.

Remark 3.3. The converse of the Theorem 3.2 need not be true. For $G=C_{5}, d(e, f) \leq 2$ for every $e, f \in E(G)$ but $\gamma_{g e e}(G)=3$.

Corollary 3.4. Let $G$ be a connected graph of size $m \geq 4$. If $d(e, f) \geq 3$ for some $e, f \in E(G)$. Then $\gamma_{g e e}(G) \geq 3$.

Theorem 3.5. Let $G$ be a connected graph with $\gamma_{g e e}(G)=2$. Then $c(G) \leq 6$, where $c(G)$ is the length of the longest cycle in $G$.

Proof. On the contrary, suppose that $c(G) \geq 7$. If $G=C_{n}(n \geq 7)$, then by Observation 2.3 (vi), $\gamma_{\text {gee }}(G) \geq 3$, which is not so. Therefore $G \neq C_{n}$ $(n \geq 7)$. Let $C v_{1}, v_{2}, v_{3}, \ldots, v_{n}, v_{1}$ be a longest cycle in $G$, where $n \geq 7$. Let $S=\{e, f\}$ be a $\gamma_{g e e}$-set of $G$. Since $G \neq C_{n}(n \geq 7)$, then exists a vertex $v$ in $G$ such that $v$ is not on $C$ and $v$ is adjacent to $v_{1}$, say. Then either $S$ is not an edge-to-edge geodetic set of $G$ or not an edge dominating set of $G$, which is a contradiction. Therefore $c(G) \leq 6$.

Theorem 3.6. If $G \neq K_{1, m}$ is a connected graph with size $m \geq 4$, then $\gamma_{\text {gee }}(G) \leq m-1$. 
Proof. If $G=K_{n}(n \geq 4)$, then by Observation $2.3(\mathrm{v}), \gamma_{\text {gee }}(G) \leq m-1$. So we have done. Therefore let us assume that $G \neq K_{n}(n \geq 4)$. Since $G \neq K_{1, m}(m \geq 4)$, then $G$ contains at least one non extreme edge, say $e$. Then $S=E(G)-\{e\}$ is an edge-to-edge geodetic dominating set of $G$ so that $\gamma_{\text {gee }}(G) \leq m-1$.

Remark 3.7. From Observation 2.3(ii), the bound in Theorem 3.6 is sharp for a double star with $m$ edges.

Theorem 3.8. Let $G$ be a connected graph of size $m \geq 4$ with diameter $d \leq 3$ which is neither a star nor a double star. Then $\gamma_{g e e}(G) \leq m-2$.

Proof. Let $G$ be a tree. Then by our assumption $G$ is not a star or a double star. Then $G$ contains at least three internal edges. Let $e_{1}, e_{2}, \ldots, e_{n}$ $(n \geq 3)$ be the internal edges of $G$. Then $S=E(G)-\left\{e_{1}, e_{n}\right\}$ is an edgeto-edge geodetic dominating set so that $\gamma_{g e e}(G) \leq m-2$.

Next we assume that $G$ is not a tree. So we have the following cases.

Case 1: $d=1$. Then $G=K_{n}$. By Observation $2.3(\mathrm{v}), \gamma_{g e e}(G) \leq m-2$.

Case 2: $d=2$. If $G=C_{n}(n \geq 4)$, then $G$ is either $C_{4}$ or $C_{5}$. By Observation 2.3 (vi), $\gamma_{\text {gee }}(G) \leq m-2$. If $G \neq C_{n}(n \geq 4)$, then $G$ contains a cycle. Since $G$ is connected and $d=2$, it follows that $c(G) \leq 4$. So we have the following two subcases.

Subcase 2a: $c(G)=4$. Let $C: v_{1}, v_{2}, v_{3}, v_{4}, v_{1}$ be a cycle in $G$. Since $G$ is not a cycle, there exists a vertex $v$ in $G$ such that $v$ is not on $C$ and $v$ is adjacent to $v_{1}$, say. Then $S=E(G)-\left\{v_{1} v_{2}, v_{1} v_{4}\right\}$ is an edge-to-edge geodetic dominating set of $G$ so that $\gamma_{\text {gee }}(G) \leq m-2$.

Subcase 2b: $c(G)=3$. Let $C: v_{1}, v_{2}, v_{3}, v_{1}$ be a cycle in $G$. Since $m \geq 4$, $G \neq C_{3}$. Then the graph is like $G$ given in Figure 3.1, which has edge-toedge geodetic domination number at most $m-2$. 

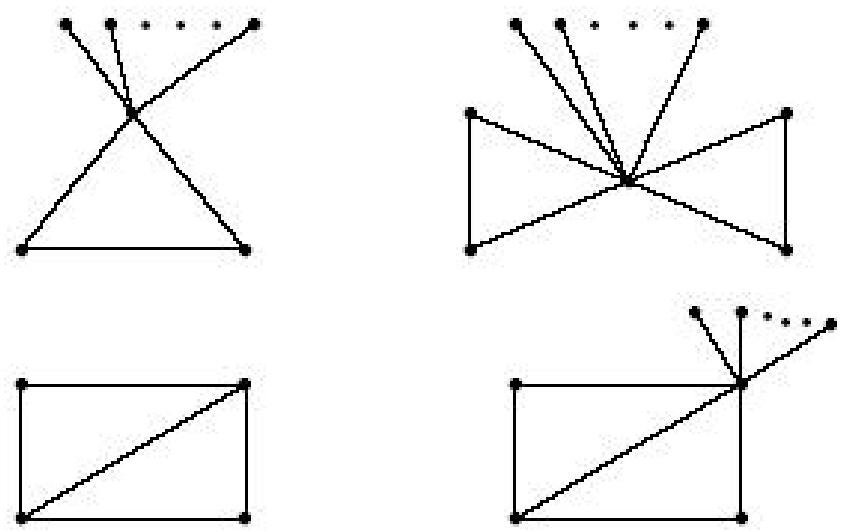

G

Figure 3.1

Case 3: $d=3$. If $G=C_{n}(n \geq 6)$, then $G=C_{6}$ or $C_{7}$. By Observation 2.3 (vi), $\gamma_{\text {gee }}(G) \leq m-2$. If $G \neq C_{n}(n \geq 6)$, since $G$ is connected and $d=3$, then $c(G) \leq 6$. We have the following four subcases.

Subcase 3a: $c(G)=6$. Let $C: v_{1}, v_{2}, v_{3}, v_{4}, v_{5}, v_{6}, v_{1}$ be a cycle in $G$. Since $G$ is not a cycle, there exists a vertex $v$ such that $v$ is not on $C$ and $v$ is adjacent to $v_{1}$, say. Without loss of generality, let us assume that $v, v_{1}, v_{2}, v_{3}$ be a diametral path in $G$. Then $S=E(G)-\left\{v_{1} v_{2}, v_{2} v_{3}\right\}$ is an edge-to-edge geodetic dominating set of $G$ and so $\gamma_{g e e}(G) \leq m-2$.

Subcase 3b: $c(G)=5$. Let $C: v_{1}, v_{2}, v_{3}, v_{4}, v_{5}, v_{1}$ be a cycle in $G$. Since $G$ is not a cycle, there exists a vertex $v$ not in $C$ and $v$ is adjacent to $v_{1}$, say. Since $d=3$, either $v$ is an end vertex of $G$ or $v$ is adjacent to a vertex, say $v_{2}$ in $G$. If $v$ is an end vertex, then $S=E(G)-\left\{v_{1} v_{2}, v_{1} v_{5}\right\}$ is an edge-to-edge geodetic dominating set of $G$ so that $\gamma_{\text {gee }}(G) \leq m-2$. If $v$ is adjacent to $v_{2}$. Then $S_{1}=E(G)-\left\{v_{4} v_{5}, v_{1} v_{5}\right\}$ is an edge-to-edge geodetic set of $G$ so that $\gamma_{g e e}(G) \leq m-2$. 
Subcase 3c: $c(G)=4$. Let $C: v_{1}, v_{2}, v_{3}, v_{4}, v_{1}$ be a cycle in $G$. Since $G \neq C_{4}$, there exists a vertex $v$ such that $v$ is not on $C$ and $v$ is adjacent to $v_{1}$, say. Without loss of generality, let $v_{4}, v_{2}, v_{1}, v$ be a diametral path in $G$. Then $S=E(G)-\left\{v_{1} v_{2}, v_{3} v_{4}\right\}$ is an edge-to-edge geodetic dominating set of $G$ so that $\gamma_{g e e}(G) \leq m-2$.

Subcase 3d: $c(G)=3$. Let $C: v_{1}, v_{2}, v_{3}, v_{1}$ be a cycle in $G$. Since $m \geq 4$, $G \neq C_{3}$. Then the graph is like $G$ given in Figure 3.2, which has an edgeto-edge geodetic domination number at most $m-2$.
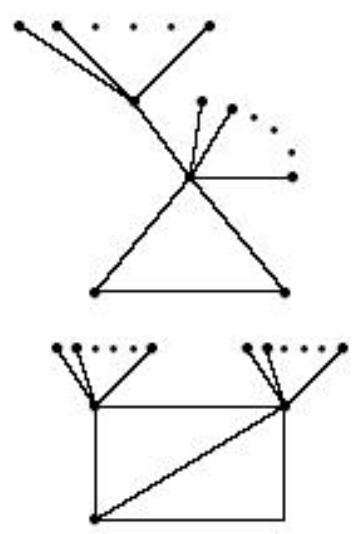
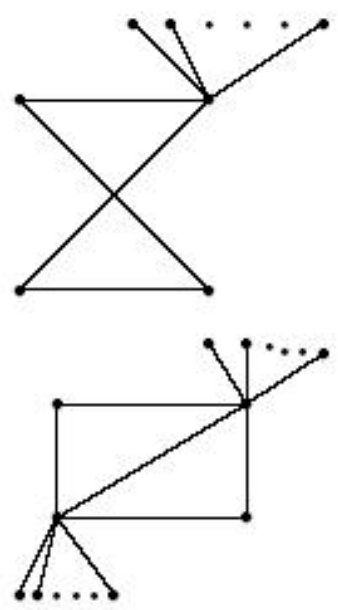

G

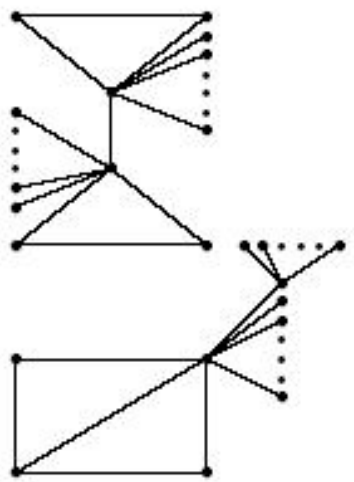

Figure 3.2

Remark 3.9. The bound in Theorem 3.8 is sharp. For the graph $G$ given in Figure 3.3, $S=E(G)-\left\{v_{1} v_{2}, v_{1} v_{3}\right\}$ is the unique $\gamma_{g e \text {-set }}$ of $G$ so that $\gamma_{\text {gee }}(G)=m-2$. 


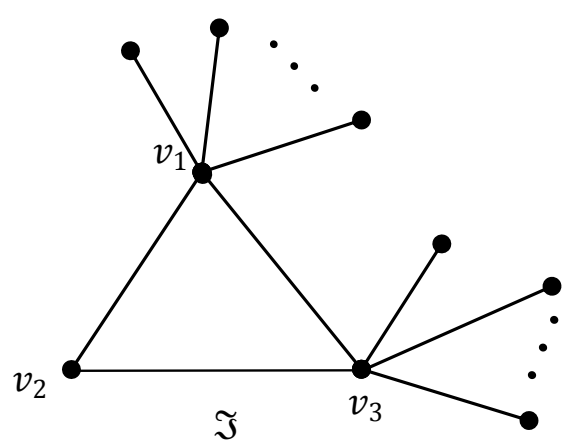

Figure 3.3

In the following theorem we give an improved upper bound for the edgeto-edge geodetic domination number of a graph in terms of its size $m$ and diameter $d$.

Theorem 3.10. If $G \neq K_{1, m}$ is a connected graph with $m \geq 4$, then $\gamma_{\text {gee }}(G) \leq m-\left\lceil\frac{2 d-3}{3}\right\rceil$, where $d$ is the diameter of $G$.

Proof. Case 1. $d \leq 3$. Since $G \neq K_{1, m}$, by Theorem 3.6, $\gamma_{g e e}(G) \leq$ $m-1 \leq m-\left\lceil\frac{2 d-3}{3}\right\rceil$.

Case 2. $d \geq 4$. Let $u$ and $v$ be vertices of $G$ for which $d(u, v)=d$, where $d$ is the diameter of $G$. Let $P: u=u_{0}, u_{1}, u_{2}, \ldots, u_{d}=v$ be a $u-v$ path of length $d$. Let $e_{i}=u_{i-1} u_{i}(1 \leq i \leq d)$.

Subcase 2a. $d \equiv 0(\bmod 3)$. Let $S=E(G)-\left\{u_{1} u_{2}, u_{2} u_{3}, u_{4} u_{5}, u_{5} u_{6}, \ldots, u_{d-2} u_{d-1}\right\}$. Then $S$ is an edge dominating set of $G$. Let $e$ be any edge of $G$. If $e=u_{i} u_{i+1}$ $(1 \leq i \leq d-1)$, then $e$ lies on the $e_{1}-e_{d}$ geodesic $P_{1}: u_{1}, u_{2}, \ldots, u_{d-1}$. If $e \neq u_{i} u_{i+1}(1 \leq i \leq d-1)$, then $e$ is an edge of $S$. Therefore, $S$ is an edgeto-edge geodetic set of $G$. Thus $S$ is an edge-to-edge geodetic dominating set of $G$. Consequently, $\gamma_{g e e}(G) \leq|S|=m-\left(\frac{2 d-3}{3}\right) \leq m-\left\lceil\frac{2 d-3}{3}\right\rceil$.

Subcase 2b. $d+1 \equiv 0(\bmod 3)$. Let $S=E(G)-\left\{u_{1} u_{2}, u_{2} u_{3}, u_{4} u_{5}, u_{5} u_{6}, \ldots, u_{d-4} u_{d-3}\right.$, $\left.u_{d-3} u_{d-2}\right\}$. Then $S$ is an edge dominating set of $G$. By the similar argument made in Subcase 2a, we get $\gamma_{\text {gee }}(G) \leq m-\left(\frac{2 d-3}{3}\right) \leq m-\left\lceil\frac{2 d-3}{3}\right\rceil$.

Subcase 2c. $d-1 \equiv 0(\bmod 3)$. Let $S=E(G)-\left\{u_{1} u_{2}, u_{2} u_{3}, u_{4} u_{5}, u_{5} u_{6}, \ldots, u_{d-3} u_{d-2}\right.$, $\left.u_{d-2} u_{d-1}\right\}$. Then $S$ is an edge dominating set of $G$. By the similar argu- 
ment made in Subcase 2a, we get $\gamma_{g e e}(G) \leq m-\left\lceil\frac{2 d-3}{3}\right\rceil$.

Remark 3.11. The bound in Theorem 3.10 is sharp. For the graph $G=$ $P_{5}, \gamma_{\text {gee }}(G)=2, m=4, d=4$ and $m-\left\lceil\frac{2 d-3}{3}\right\rceil=2$. Therefore $\gamma_{\text {gee }}(G)=$ $m-\left\lceil\frac{2 d-3}{3}\right\rceil$. Also the bound in Theorem 3.10 can be strict. For the graph $G$ given in Figure 3.4, $\gamma_{\text {gee }}(G)=3, m=9, d=5$ and $m-\left\lceil\frac{2 d-3}{3}\right\rceil=6$. Thus $\gamma_{\text {gee }}(G)<m-\left\lceil\frac{2 d-3}{3}\right\rceil$.

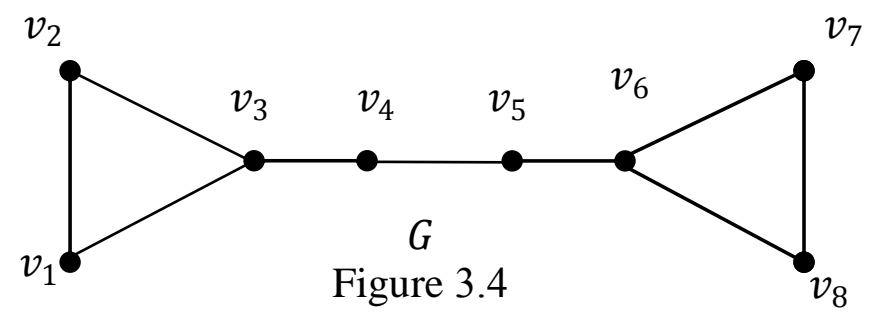

\section{Graph with small and large edge-to-edge geodetic domi- nation number}

In this section, we characterized connected graphs $G$ of size $m$ with $\gamma_{\text {gee }}(G)=2$ or $m$ or $m-1$. For this purpose we introduce the collection $\Im$ of graphs given in Figure 4.1. 
Figure 4.1.

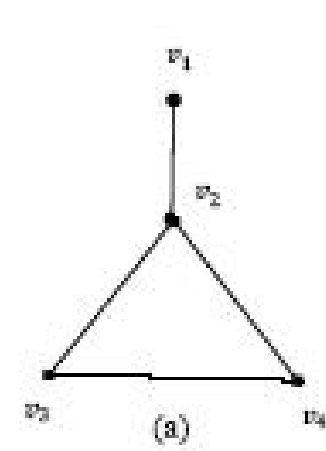

(a)

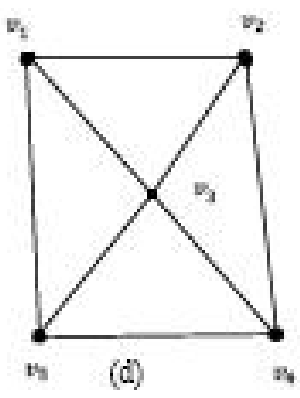

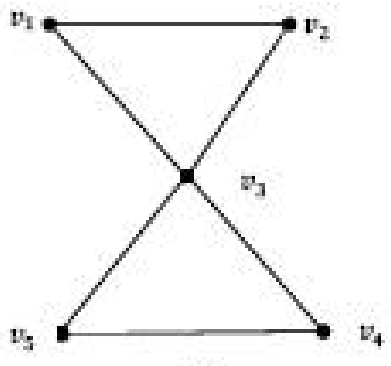

(c)
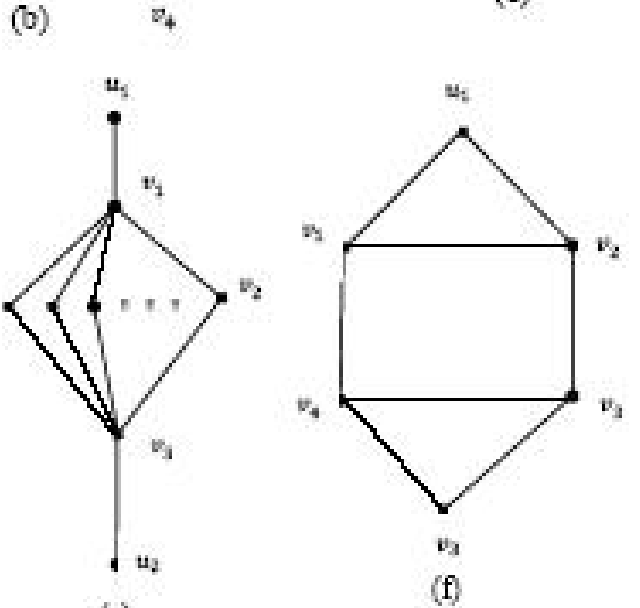

(e)

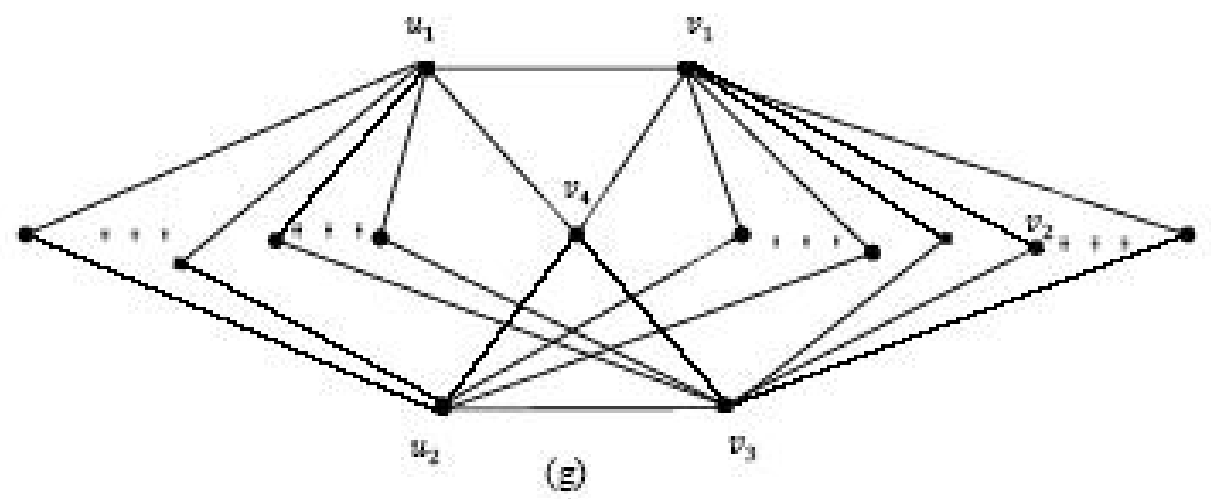



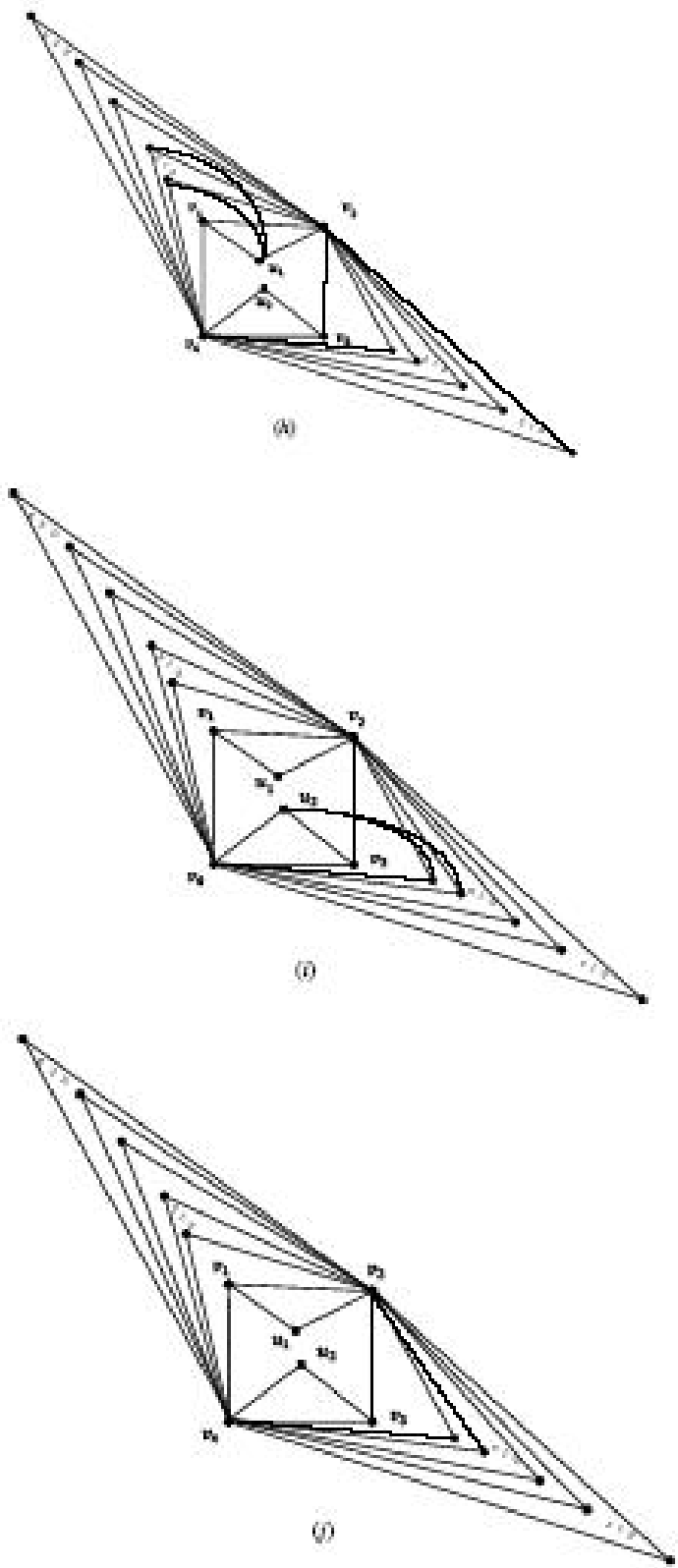


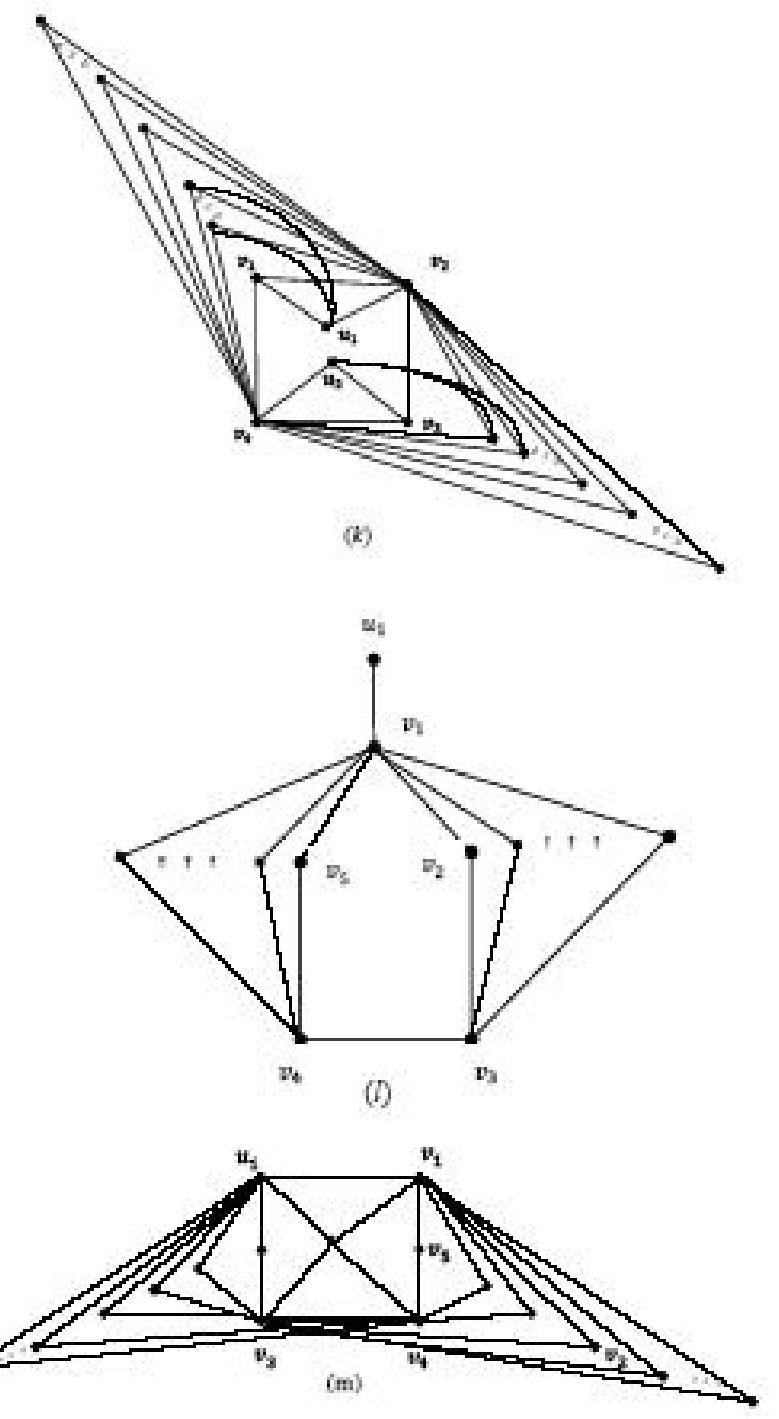



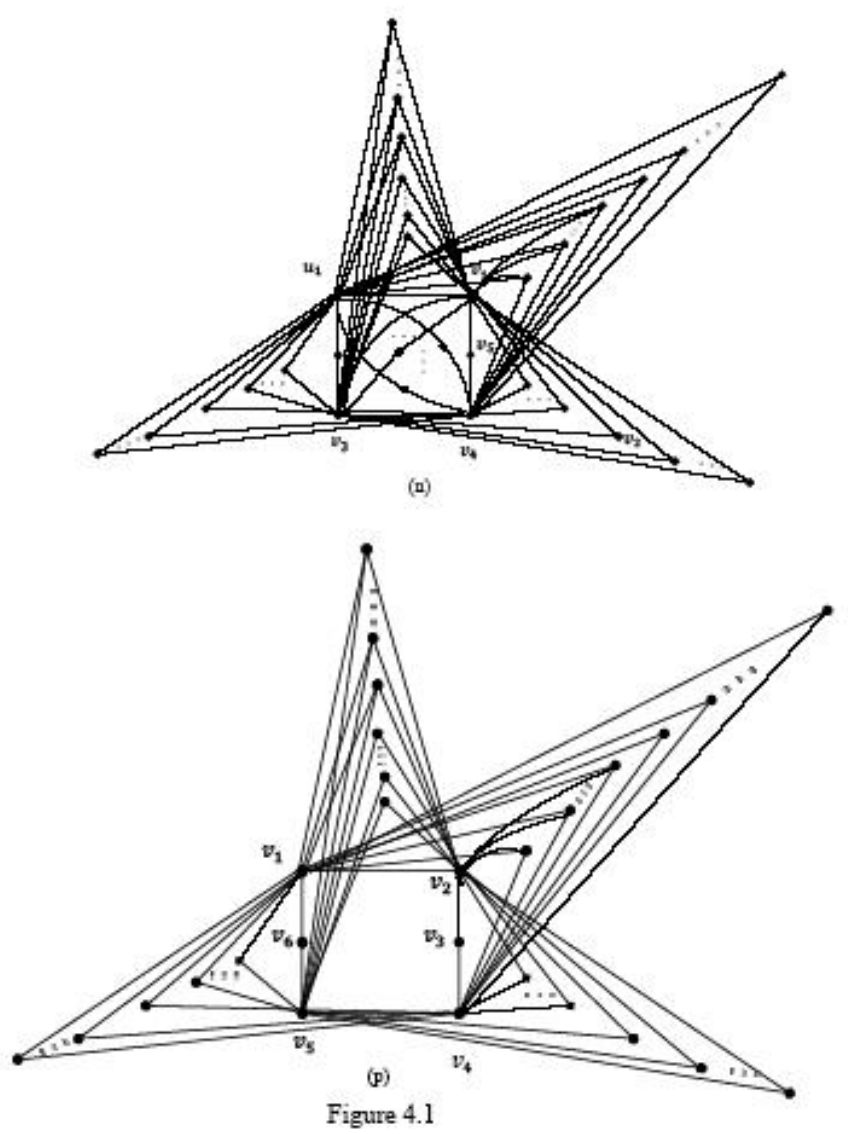

Theorem 4.1. Let $G$ be a connected graph with size $m \geq 2$. Then $\gamma_{g e e}(G)=2$ if and only if $G$ is either $P_{3}$ or $P_{4}$ or $P_{5}$ or $C_{4}$ or $K_{4}-\{e\}$ or $K_{4}$ or $C_{6}$ or the class of graphs $\Im$ given in Figure 4.1. 
Proof. Let $\gamma_{g e e}(G)=2$. Then by Theorem 3.2, $d(e, f) \leq 2$ for every $e, f \in E(G)$. If $G$ is a tree, then $G=P_{3}$ or $P_{4}$ or $P_{5}$. Which satisfies the requirements of the theorem. Let us assume that $G$ is not a tree. Let $c(G)$ be the length of a longest cycle in $G$. By Theorem 3.5, $c(G) \leq 6$. Let $C$ be a longest cycle in $G$. We consider four cases.

Case 1. $c(G)=3$. Let $C$ be $v_{1}, v_{2}, v_{3}, v_{1}$. First assume that $G$ contains only one cycle $C$ with $|C|=3$. If $G=C_{3}$, then $\gamma_{\text {gee }}(G)=3>2$, which is a contradiction. Therefore $G \neq C_{3}$. Then there exists a vertex $u_{1}$ not in $C$ and $u_{1}$ is adjacent to $v_{1}$, say. Since $G$ contains only one cycle, $v_{1}$ is a cut vertex of $G$. Since $\gamma_{\text {gee }}(G)=2$, there exist only two components, say $G_{1}$ and $G_{2}$ of $G-v_{1}$ such that $\left|V\left(G_{1}\right)\right| \leq 2$ and $\left|V\left(G_{2}\right)\right| \leq 2$. Since $G$ is not a tree, $\left|V\left(G_{1}\right)\right|=\left|V\left(G_{2}\right)\right| \neq 1$. If $\left|V\left(G_{1}\right)\right|=1$ and $\left|V\left(G_{2}\right)\right|=2$, then the graph $G$ is given in class of graphs $\Im$ of Figure 4.1 (a), which satisfies the requirements of the theorem. If $\left|V\left(G_{1}\right)\right|=\left|V\left(G_{2}\right)\right|=2$, then the graph $G$ is given in class of graphs $\Im$ of Figure 4.1 (b), which satisfies the requirements of the theorem. Suppose that $G$ contains another one cycle $C_{1}$ with $|C|=\left|C_{1}\right|=3$. If $C$ and $C_{1}$ have a common vertex, then the graph $G$ is given in the class of graphs $\Im$ of Figure 4.1(c,d), which satisfies the requirements of the theorem. If $C$ and $C_{1}$ have a common edge, then the graph $G$ is either $K_{4}-\{e\}$ or $K_{4}$, which satisfies the requirements of the theorem. If $C$ and $C_{1}$ is connected by a path $P: x, x_{1}, x_{2}, \ldots, x_{n}=y$, where $x \in V(C)$ and $y \in V\left(C_{1}\right)$ and $n \geq 1$. Then $d(e, f) \geq 3$ for some $e, f \in E(G)$ and so $\gamma_{g e e}(G) \geq 3$, which is a contradiction.

Case 2. $c(G)=4$. Let $C$ be $v_{1}, v_{2}, v_{3}, v_{4}, v_{1}$. If $G=C_{4}$, then $G$ satisfies the requirements of the theorem. If $G \neq C_{4}$, then there exists a vertex $u_{1}$ not in $C$ and $u_{1}$ is adjacent to $v_{1}$, say. Since $\gamma_{\text {gee }}(G)=2$, there exists a vertex $u_{2}$ not in $C$ and $u_{2}$ is adjacent to $v_{3}$. If $\operatorname{deg}\left(u_{1}\right)=1$ and $\operatorname{deg}\left(u_{2}\right)=1$, then the graph $G$ is given in class of graphs $\Im$ of Figure 4.1(e). If $\operatorname{deg}\left(u_{1}\right)=2$ and $\operatorname{deg}\left(u_{2}\right)=2$, then the graph $G$ is given in class of graphs $\Im$ of Figure 4.1(f). If $\operatorname{deg}\left(u_{1}\right)=1$ and $\operatorname{deg}\left(u_{2}\right)=2$ or if $\operatorname{deg}\left(u_{1}\right)=2$ and $\operatorname{deg}\left(u_{2}\right)=1$, then $\gamma_{\text {gee }}(G) \geq 3$, which is not so. If $\operatorname{deg}\left(u_{1}\right) \geq 2$ and $\operatorname{deg}\left(u_{2}\right) \geq 3$ or if $\operatorname{deg}\left(u_{1}\right) \geq 3$ and $\operatorname{deg}\left(u_{2}\right) \geq 2$, and also since $\gamma_{\text {gee }}(G)=2$, then there must be at least one $u_{1} v_{1}-u_{2} v_{3}$ geodesic. Then the graph $G$ given in class of graphs $\Im$ of Figure $4.1(g, h, i, j, k)$. Note that $S=\left\{u_{1} v_{1}, u_{2} v_{3}\right\}$ is a $\gamma_{g e e}$-set of $G$ so that $\gamma_{g e e}(G)=2, G$ satisfies the requirements of the theorem.

Case 3. $c(G)=5$. Let $C$ be $v_{1}, v_{2}, v_{3}, v_{4}, v_{5}, v_{1}$. If $G=C_{5}$, then $\gamma_{g e e}(G)=$ 
3 , which is a contradiction. Therefore $G \neq C_{5}$. Then there exists a vertex $u_{1}$ not in $C$ and $u_{1}$ is adjacent to $v_{1}$, say. If $\operatorname{deg}\left(u_{1}\right)=1$, then since $\gamma_{g e e}(G)=2$. their must be at least one $u_{1} v_{1}-u_{3} v_{4}$ geodesic. Then the graph $G$ given in class of graphs $\Im$ of Figure 4.1(1). Note that $S=\left\{u_{1} v_{1}, u_{3} v_{4}\right\}$ is a $\gamma_{g e e}$-set of $G$ so that $\gamma_{g e e}(G)=2$. Therefore $G$ satisfies the requirements of the theorem. If $\operatorname{deg}\left(u_{1}\right) \geq 2$, then there must be at least one $u_{1}-v_{3}$ geodesic or $u_{1}-v_{4}$ geodesic or $v_{1}-v_{4}$ geodesic or $v_{1}-v_{3}$ geodesic or $u_{1}-v_{3}$. Then the graph $G$ given in class of graphs $\Im$ of Figure 4.1(m,n). Note that $S=\left\{u_{1} v_{1}, v_{3} v_{4}\right\}$ is a $\gamma_{g e e}$-set of $G$ so that $\gamma_{g e e}(G)=2$, which $G$ satisfies the requirements of the theorem.

$\Im$ of Figure 4.1(g), which satisfies the requirements of the theorem. If $\operatorname{deg}(v) \geq 2$, since $d(e, f) \leq 2$ for every $e, f \in E(G)$, then there must be a path $P$ of length three which joins two non adjacent vertices of $C$. Let the path $P$ be $v_{1}, u_{2}, u_{3}, v_{4}$, where $u_{2} \neq v_{2}$ and $u_{3} \neq v_{3}$. Then $\gamma_{g e e}(G)=2$. Therefore the graph $G$ is given in class of graphs $\Im$ of Figure 4.1 (h), which satisfies the requirements of the theorem. If $v_{2} v_{5} \in E(G)$, then $\gamma_{g e e}(G)=2$. Therefore the graph $G$ is given in class of graphs $\Im$ of Figure 4.1(i), which satisfies the requirements of the theorem.

Case 4. $c(G)=6$. Let $C$ be $v_{1}, v_{2}, v_{3}, v_{4}, v_{5}, v_{6}, v_{1}$. If $G=C_{6}$, then $G$ satisfies the requirement of the theorem. If $G \neq C_{6}$, there exist at least two $v_{1} v_{2}-v_{4} v_{5}$ geodesics. Then the graph $G$ given in class of graphs $\Im$ of Figure 4.1(p). Note that $S=\left\{v_{1} v_{2}, v_{4} v_{5}\right\}$ is a $\gamma_{g e e}$-set of $G$ so that $\gamma_{g e e}(G)=2$, which $G$ satisfies the requirements of the theorem.

The converse is clear.

Theorem 4.2. Let $G$ be a connected graph of size $m \geq 2$. Then $\gamma_{\text {gee }}(G)=$ $m$ if and only if $G$ is the star $K_{1, m}$ or $K_{3}$.

Proof. Let $G=K_{1, m}(m \geq 2)$. Then by Observation 2.3 (ii), $\gamma_{g e e}(G)=$ $m$. If $G=K_{3}$, then $S=E(G)$ is the unique $\gamma_{\text {gee }}$-set of $G$ so that $\gamma_{g e e}(G)=$ $3=m$. For the converse, let $G$ be a connected graph of size $m \geq 2$ and $\gamma_{g e e}(G)=m$. Then by Theorem 3.10, $d \leq 2$. If $d=1$, then $G=K_{n}$ $(n \geq 3)$. If $n \geq 4$, then by Observation $2.3(\mathrm{v}), \gamma_{\text {gee }}(G) \leq m-1$, which is not so. Therefore $m=3$. Which implies $G=K_{3}$, which satisfies the requirements of the theorem. Let $d=2$. Let $m=3$. If $G$ is a tree, then $G=K_{1,3}$, which satisfies the requirement of this theorem. If $G$ is not a tree, then $G=K_{3}$, which satisfies the requirements of this theorem. Let $m=2$. Then $G=K_{1,2}$, which satisfies the requirements of this theorem. 
Let $m \geq 4$. If $G \neq K_{1, m}$, then by Theorem 3.6, $\gamma_{\text {gee }}(G) \leq m-1$, which is a contradiction. Therefore $G=K_{1, m}$. First assume that $m \geq 4$. Therefore $G=K_{3}$ or $G=K_{1, m}$.

Theorem 4.3. Let $G$ be a connected graph of size $m \geq 3$. Then $\gamma_{g e e}(G)=$ $m-1$ if and only if $G$ is a double star.

Proof. If $G$ is a double star, then by Observation 2.3(iii), $\gamma_{g e e}(G)=$ $m-1$. For the converse, let $G$ be connected graph of size $m \geq 3$ and $\gamma_{\text {gee }}(G)=m-1$. Let $m=3$. If $G$ is a tree, then $G=P_{4}$ or $K_{1,3}$. If $G=P_{4}$, then $\gamma_{\text {gee }}(G)=2=m-1$, which satisfies the requirement of the theorem. If $G=K_{1,3}$, then $G$ is a star. By Theorem 4.2, $\gamma_{\text {gee }}(G)=3=m$, which is a contradiction. If $G$ is not a tree, then $G=K_{3}$. By Theorem 4.2, $\gamma_{\text {gee }}(G)=3=m$, which is a contradiction. Let $m \geq 4$. Then by Theorem $3.10, m-1 \leq\left\lceil\frac{2 d-3}{3}\right\rceil$

$\Rightarrow \quad d \leq 3$

If $G$ is neither a star nor a double star, then by Theorem 3.8, $\gamma_{g e e}(G) \leq$ $m-2$, which is a contradiction. Therefore $G$ is either a star or a double star. If $G$ is a star, then by Theorem $4.2, \gamma_{\text {gee }}(G)=m$, which is a contradiction. If $G$ is a double star, then by Observation 2.3 (iii), $\gamma_{\text {gee }}(G)=m-1$, which satisfies the requirements of the theorem.

In the following, we present the Nordhaus-Gaddum type relations for edge-to-edge geodetic dominating number of a graph.

Theorem 4.4. If $G$ and $\bar{G}$ are connected graphs of size $m \geq 4$, then $4 \leq \gamma_{\text {gee }}(G)+\gamma_{\text {gee }}(\bar{G}) \leq 2 m-2$. Moreover the lower and bound are sharp if and only if $G=P_{4}$.

Proof. $\quad$ Since $G$ and $\bar{G}$ are connected graphs, $\gamma_{\text {gee }}(G) \geq 2$ and $\gamma_{g e e}(\bar{G}) \geq$ 2 and so $\gamma_{\text {gee }}(G)+\gamma_{\text {gee }}(\bar{G}) \geq 4$. Since $\bar{G}$ is connected, by Theorem 4.2 , $\gamma_{e e}(\bar{G}) \leq m-1$. Which implies $\gamma_{e e}(\bar{G}) \leq m-1$. Hence $\gamma_{g e e}(G)+\gamma_{g e e}(\bar{G}) \leq$ $2 m-2$. Therefore $4 \leq \gamma_{\text {gee }}(G)+\gamma_{\text {gee }}(\bar{G}) \leq 2 m-2$. Next we prove that $\gamma_{\text {gee }}(G)+\gamma_{\text {gee }}(\bar{G})=4$ if and only if $G=P_{4}$. From Theorem 4.1, we can easily verified that $\gamma_{\text {gee }}(G)=2$ and $\gamma_{g e e}(\bar{G})=2$ if and only if $G=P_{4}$. Therefore $\gamma_{\text {gee }}(G)+\gamma_{\text {gee }}(\bar{G})=4$ if and only if $G=P_{4}$. Next we prove that $\gamma_{g e e}(G)+\gamma_{g e e}(\bar{G})=2 m-2$ if and only if $G=P_{4}$. By Theorem 4.3, $\gamma_{g e e}(G)=m-1$ and $\gamma_{e e}(\bar{G})=m-1$ if and only if $G$ is a double star. For a double star $G=\bar{G}$ if and only if $m=3$. Therefore $\gamma_{\text {gee }}(G)=m-1$ and $\gamma_{\text {gee }}(\bar{G})=m-1$ if and only if $G=P_{4}$. Hence $\gamma_{\text {gee }}(G)+\gamma_{\text {gee }}(\bar{G})=2 m-2$ if and only if $G=P_{4}$. 


\section{Realization Results}

In this section we give some realization results concerning the edge-toedge geodetic domination number. Further, we will also make use of the following Theorem 5.1, which is proved in [1].

Theorem 5.1. [1] Each end edge of $G$ belongs to every edge-to-edge geodetic set of $G$.

Theorem 5.2. For any integer $a \geq 2$, there exists a connected graph $G$ with $\gamma_{e}(G)=g_{e e}(G)=\gamma_{g e e}(G)=a$.

Proof. Let $P_{i}: u_{i}, v_{i}(1 \leq i \leq a)$ be a copy of path on two vertices. Let $G$ be a graph obtained from $P_{i}(1 \leq i \leq a)$ by adding a new vertex $x$ and join $x$ with each $u_{i}(1 \leq i \leq a)$ and with each $v_{i}(1 \leq i \leq a)$. The graph $G$ is shown in Figure 5.1.

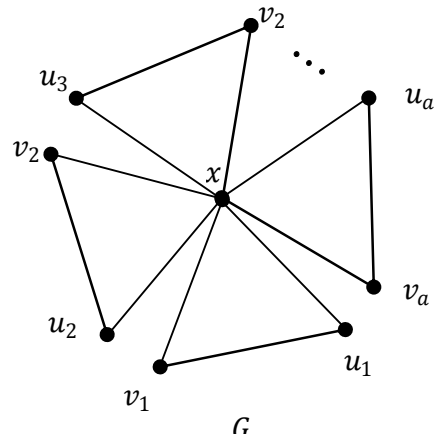

Figure 5.1

First we claim that $\gamma_{e}(G)=a$. Let $H_{i}=\left\{x u_{i}, x v_{i}, u_{i} v_{i}\right\}(1 \leq i \leq$ a). It is easily observed that every edge dominating set of $G$ contains at least one edge from each $H_{i}(1 \leq i \leq a)$ and so $\gamma_{e}(G) \geq a$. Now $S=$ $\left\{u_{1} v_{1}, u_{2} v_{2}, \ldots, u_{a} v_{a}\right\}$ is an edge dominating set of $G$ so that $\gamma_{e}(G)=a$. Next we show that $g_{e e}(G)=a$. It is easily observed that every $g_{e e}$-set of $G$ contains only the edge $u_{i} v_{i}$ of $H_{i}(1 \leq i \leq a)$ and so $g_{e e}(G) \geq a$. Now $S$ is an edge-to-edge geodetic set of $G$ so that $g_{e e}(G)=a$. Next we show that $\gamma_{g e e}(G)=a$. Since $S$ is both a $\gamma_{e}$-set of $G$ and a $g_{e e^{-s e t}}$ of $G, S$ is a $\gamma_{g e e}$-set of $G$ so that $\gamma_{g e e}(G)=a$.

Theorem 5.3. For every pair of positive integers $a$, $b$ with $2 \leq a \leq b$, there exists a connected graph $G$ with $g_{e e}(G)=a$ and $\gamma_{g e e}(G)=b$. 


\section{Proof.}

For $2 \leq a=b$, the result from Theorem 5.2. Therefore let $2 \leq a<b$. Let $P_{i}: u_{i}, v_{i}(1 \leq i \leq b-a)$ be a copy path of order 2 . Let $G$ be a graph obtained from $P_{i}(1 \leq i \leq b-a)$ by adding new vertices $x, y, z$ and $z_{1}, z_{2}, \ldots, z_{a-1}$ and joining $x$ with $y, y$ with each $u_{i}(1 \leq i \leq b-a)$, $z$ with each $v_{i}(1 \leq i \leq b-a)$ and $z$ with each $z_{i}(1 \leq i \leq a-1)$. The graph $G$ is shown in Figure 5.2.

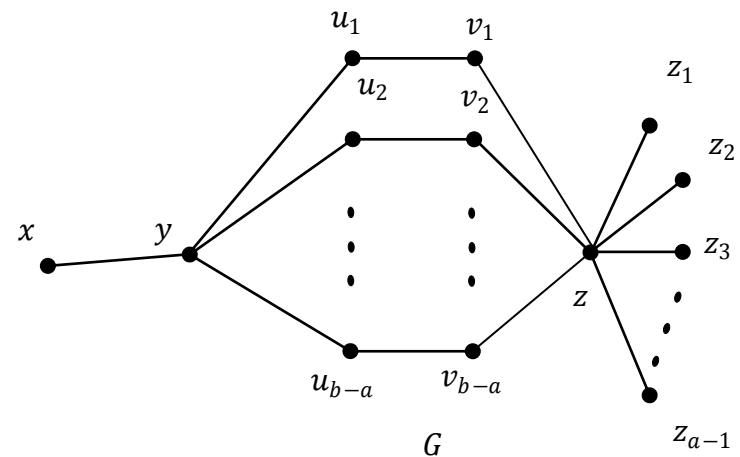

Figure 5.2

First we claim that $g_{e e}(G)=a$. Let $X=\left\{x y, z z_{1}, z z_{2}, \ldots, z z_{a-1}\right\}$ be the set of all end edges of $G$. By Theorem 5.1, $X$ is a subset of every edge-to-edge geodetic set of $G$ and so $g_{e e}(G) \geq a$. It is clear that $X$ is an edge-to-edge geodetic set of $G$ so that $g_{e e}(G)=a$.

Next we show that $\gamma_{g_{e e}}(G)=b$. By Observation 2.3(i), $X$ is a subset of every edge-to-edge geodetic dominating set of $G$. For $1 \leq i \leq b-a$, let $H_{i}=\left\{y u_{i}, u_{i} v_{i}, v_{i} z\right\}$. Then it is observed that every edge-to-edge geodetic dominating set of $G$ contains at least one edge from each $H_{i}(1 \leq i \leq b-a)$ and so $\gamma_{g e e}(G) \geq b$. Let $Y=\left\{u_{1} v_{1}, u_{2} v_{2}, \ldots, u_{b-a} v_{b-a}\right\}$. Then $S=X \cup Y$ is an edge-to-edge geodetic dominating set of $G$ so that $\gamma_{g e e}(G)=b$.

Theorem 5.4. For every pair of positive integers $a$, $b$ with $2<a \leq b$, there exists a connected graph $G$ with $\gamma_{e}(G)=a$ and $\gamma_{g e e}(G)=b$.

Proof. For $2 \leq a=b$, the result from Theorem 5.2. Therefore let $2 \leq a<b$. Let $Q_{i}: u_{i}, v_{i}, w_{i}, x_{i}(1 \leq i \leq a-2)$ be a copy of path on four vertices. Let $G$ be a graph obtained from $Q_{i}(1 \leq i \leq a-2)$ by adding new vertices $p, q, r, s, z_{1}, z_{2}, \ldots, z_{b-a}$ and join $p$ with $q, q$ with each $u_{i}(1 \leq i \leq a-2), r$ with each $x_{i}(1 \leq i \leq a-2), r$ with $s$ and $s$ with each $z_{i}(1 \leq i \leq b-a)$. The graph $G$ is shown in Figure 5.3. 


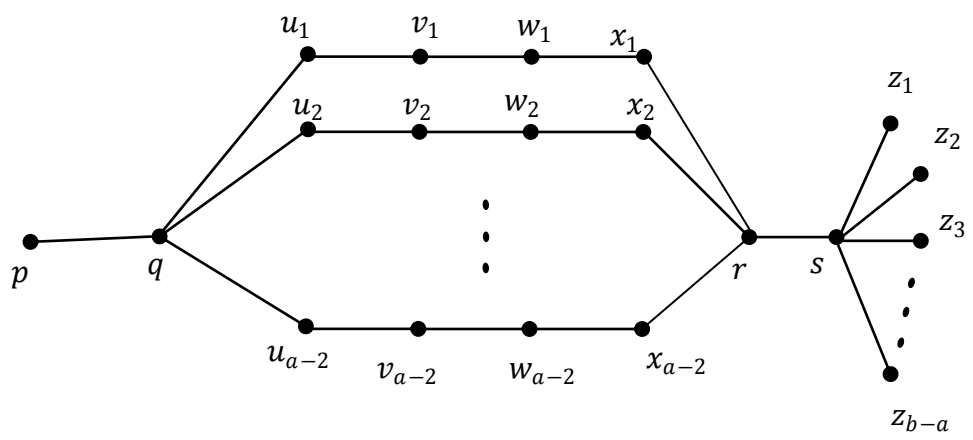

$G$

Figure 5.3

First we claim that $\gamma_{e}(G)=a$. It is easily observed that every minimum edge dominating set of $G$ contains the edges $v_{i} w_{i}(1 \leq i \leq a-2)$ and so $\gamma_{e}(G) \geq a-2$. Let $S=\left\{v_{1} w_{1}, v_{2} w_{2}, \ldots, v_{a-2} w_{a-2}\right\}$. Then $S$ is not an edge dominating set of $G$ and so $\gamma_{e}(G) \geq a-1$. It is easily verified that $S \cup\{f\}$, where $f \notin S$ is not an edge dominating set of $G$ and $\gamma_{e}(G) \geq a$. Let $S_{1}=S \cup\{p q, r s\}$. Then $S_{1}$ is the edge dominating set of $G$ so that $\gamma_{e}(G)=a$. Next we show that $\gamma_{g e e}(G)=b$. Let $X=\left\{p q, s z_{1}, s z_{2}, \ldots, s z_{b-a}\right\}$ be the set of all end edges of $G$. By Observation 2.3(i), $X$ is a subset of every edge-to-edge geodetic dominating set of $G$ and so $\gamma_{g e e}(G) \geq b-a+1$. It is easily observed that every minimum edge-to-edge gedetic dominating set of $G$ contains the edges $v_{i} w_{i}(1 \leq i \leq a-2)$ and so $\gamma_{g e e}(G) \geq b-1$. Let $Z=\left\{v_{1} w_{1}, v_{2} w_{2}, \ldots, v_{a-2} w_{a-2}\right\}$ and $M=S \cup Z$. Then $M$ is not an edgeto-edge geodetic dominating set of $G$ and so $\gamma_{\text {gee }}(G) \geq b$. Now $M \cup\{r s\}$ is an edge-to-edge geodetic dominating set of $G$ so that $\gamma_{g e e}(G)=b$.

\section{Conclusion}

This paper introduces a new parameter known as edge-to-edge geodetic domination number of a graph. Further, this concept can be extended to conditional parameters.

\section{Acknowledgment}

The authors express their gratitude to the referees for the useful suggestions. 


\section{References}

[1] M. Antony and A. L. Merlin Sheela, "The edge-to-edge geodetic number of a graph", Journal of applied science and computation, vol. 6, no. 5, pp. 1954-1962, 2019.[On line]. Available: https:/ / bit.ly/ 2Qd6r71

[2] S. Arumugam and S. Velammal, "Edge domination in graphs", Taiwanese journal of mathematics, vol. 2, no. 2, pp. 173-179, 1998, doi: $10.11650 /$ twjm/ 1500406930

[3] B. Xu, "On edge domination number of graphs", Discrete mathematics, vol. 294, no. 3, pp. 311-316, 2005, doi: 10.1016/j.disc.2004.11.008

[4] S. Beulah Samli and S. Robinson Chellathurai, "Geo chromatic number of a graph", International journal of scientific research in mathematical and statistical sciences, vol. 5, no. 6, pp. 259-264, 2018, doi: 10.26438/ ijsrmss/ v5i6.259264

[5] S. Beulah Samli. J. John , and S. Robinson Chellathurai, "The double geo chromatic number of a graph", Bulletin of the International Mathematical Virtual Institute, vol. 11, no. 1, pp. 25-38, 2021. [On line]. Available: https:/ / bit.ly/ 3v16C3Y

[6] G. Chartrand, F. Harary, and P. Zhang, "On the geodetic number of a graph", Networks, vol. 39, no. 1, pp. 1-6, 2002, doi: 10.1002/ net.10007

[7] G. Chartrand, E. M. Palmer, and P. Zhang, "The geodetic number of a graph: a survey”, Congressus numerantium, vol. 156, pp. 37-58, 2002.

[8] R. D. Dutton and R. C. Brigham, "An external problem for edge domination insensitive graphs", Discrete applied mathematics, vol. 20, no. 3, pp. 113-125, 1988, doi: 10.1016/ 0166-218X(88)90058-3

[9] T. W. Haynes, S. T. Hedetniemi, and P. J. Slater, Fundamentals of domination in graphs. New York, NY: M. Dekker, 1998.

[10] A. Hansberg and L. Volkmann, "On the geodetic and geodetic domination numbers of a graph", Discrete mathematics, vol. 310, no. 15-16, pp. 2140-2146, 2010, doi: 10.1016/j.disc.2010.04.013

[11] J. John, G. Edwin, and P. A. P. Sudhahar, "The Steiner domination number of a graph", International journal of mathematics and computer applications research, vol. 3, no. 3, pp. 37-42, 2013. [On line]. Available: https:/ / bit.ly/ 3uXvauQ 
[12] J. John and N. Arianayagam, "The detour domination number of a graph", Discrete mathematics, algorithms and applications, vol. 9, no. 1, Art. ID. 1750006, 2017, doi: 10.1142/ S1793830917500069

[13] J. John, P. A. P. Sudhahar, and D. Stalin, "On the (M, D) number of a graph", Proyecciones (Antofagasta), vol. 38, no. 2, pp. 255-266, 2019, doi: 10.4067/ S0716-09172019000200255

[14] J. John and D. Stalin, "Edge geodetic self-decomposition in graphs", Discrete mathematics, algorithms and applications, vol. 12, no. 5, Art. ID. 2050064,2020, doi: 10.1142/ S1793830920500640

[15] J. John and D. Stalin, "The edge geodetic self decomposition number of a graph", RAIRO-Operations Research, vol. 55, 2021, doi: 10.1051/ro/ 2020073

[16] S. Mitchell and S. T. Hedetniemi, "Edge domination in trees", Congressus numerantium, vol. 19, pp. 489-509, 1977.

[17] M. N. S. Paspasan and S. R. Canoy Jr, "Edge domination and total edge domination in the join of graphs", Applied mathematical science, vol. 10, no. 21, pp. 1077-1086, 2016, doi: 10.12988/ AMS.2016.6130

[18] M. N. S. Paspasan and S. R. Canoy Jr, "Edge domination in lexico and cartesian products of graphs", Far east journal of mathematical science, vol. 102, no. 2, pp. 337-347, 2017, doi: 10.17654/ MS1 02020337

[19] M. Hajian, M. A. Henning, and N. J. Rad, "A new lower bound on the domination number of a graph", Journal of combinatorial optimization, vol. 38, no. 3, pp. 721-738, 2019, doi: 10.1007/ s10878-019-00409-x

[20] P. Gupta, "Domination in graph with application", Paripex indian journal of research, vol. 2, no. 3, pp. 115-117, 2013. [On line]. Available: https:/ / bit.ly/ 3x7pEru

[21] A. P. Santhakumaran and J.John, "On the edge-to-vertex geodetic number of a graph", Miskolc mathematical notes, vol. 13, no. 1, pp. 107-119, 2012, doi: 10.18514/ MMN.2012.353

[22] A. P. Santhakumaran and J. John, "The upper connected edge geodetic number of a graph", Filomat, vol. 26, no. 1, pp. 131-141, 2012, doi: 10.2298/ FIL1201131S

[23] D. Stalin and J. John, "Edge geodetic dominations in graphs", International journal of pure and applied mathematics, vol. 116, no. 22, pp. 31-40, 2017. [On line]. Available: https:/ / bit.ly/ 3dtlDpB 
[24] S. Sujitha, J. John, and A. Vijayan, "Extreme edge-to-vertex geodesic graphs", International journal of mathematics research, vol. 6, no. 3, pp. 279-288, 2014.[On line]. Available: https:/ / bit.ly/ 3srbXjr

[25] S. Sujitha, J. John, and A. Vijayan, "The forcing edge-to-vertex geodetic number of a graph", International journal of pure and applied mathematics, vol. 103, no. 1, 2015, pp. 109-121, 2015, doi: 10.12732/ ijpam.v103i1.9

[26] V. R. Sunil Kumar and J. John, "The total detour domination number of a graph", Infokara research, vol. 8, no. 8, pp. 434-440, 2019. [On line]. Available: https:/ / bit.ly/ 3mWBFeJ

[27] F-H. Wang , Y-L. Wang, and J-M. Chang, "The lower and upper forcing geodetic numbers of blockcactus graphs", European journal of operational research, vol. 175, no. 1, pp. 238-245, 2006, doi: 10.1016/ j.ejor.2005.04.026

[28] M. Yannakakis and F. Gavril, "Edge dominating sets in graphs", SIAM journal on applied mathematics, vol. 38, no. 3, pp. 364-372, 1980, doi: $10.1137 / 0138030$ 